\title{
Synthesis of benzoazole ionic liquids and evaluation of their antimicrobial activity
}

\section{Síntese de líquidos iónicos derivados de benzoazóis e avaliação da sua atividade antimicrobiana}

Lukasz Czekański', Tânia Santos de Almeida ${ }^{2,3}$, Joana P. Mota ${ }^{2}$, Patrícia Rijo ${ }^{2}$, Maria Eduarda M. Araújo ${ }^{3}$

${ }^{1}$ Faculty of Chemistry, Laboratory of Chemistry of Heterocyclic Compounds, Adam Mickiewicz University, ul. Umultowska 89b,61-614 Poznań, Poland

${ }^{2}$ CBios - Research Center for Biosciences and Health Technologies, U Lusófona, Campo Grande 376, 1749-024 Lisboa, Portugal

${ }^{3}$ Centro de Química e Bioquímica, Faculdade de Ciências, Universidade de Lisboa, Campo Grande, 1749-016 Lisboa, Portugal, and Departamento de Química e Bioquímica, Faculdade de Ciências, Universidade de Lisboa, Campo Grande, 1749-

016 Lisboa, Portugal

E-mail: eduaraujo@fc.ul.pt

This work was funded by FCT- Fundação para a Ciência e Tecnologia (Portugal) - project PEst-OE/QUI/ UI0612/2013

Este trabalho foi financiado pela FCT-Fundação para a Ciência e Tecnologia (Portugal) através do projecto PEstOE/QUI/UI0612/2013

\begin{abstract}
A new class of chemicals composed not of molecules but ions, an organic cation and an organic or inorganic anion, has recently attracted attention. When these new kind of salts are in the liquid state below $100 \mathrm{oC}$, they are named ionic liquids (ILs). In this work, the synthesis of ionic liquids obtained from a 2-mercaptobenzimidazole, 2-mercaptobenzoxazole or 2-mercaptobenzothiazole anion and 1-alkyl-3-methylimidazolium or choline cation are described. The antimicrobial activity against several Gram-positive and Gram-negative bacteria and a yeast was also evaluated.
\end{abstract}

Keywords: Ionic liquids, benzoazole, choline, antimicrobial activity

\section{Resumo:}

Uma nova classe de compostos químicos constituídos não por moléculas mas por iões, um catião orgânico e um anião orgânico ou inorgânico, tem atraído recentemente muita atenção. Quando estes novos tipos de sais estão no estado líquido, abaixo de $100^{\circ} \mathrm{C}$, são chamados líquidos iónicos (LI). Neste trabalho, é descrita a síntese de líquidos iónicos obtidos a partir de 2-mercaptobenzimidazol, 2-mercaptobenzoxazol ou o anião 2-mercaptobenzotiazol e o catião 1-alquil-3-metilimidazólio ou o catião colina. A atividade antimicrobiana destes LI sobre várias bactérias Gram-positivas, Gram-negativas e uma levedura foi avaliada.

Palavras-chave: Líquidos iónicos, benzoazol, colina, actividade antimicrobiana 


\section{Introduction}

Ionic Liquids (ILs) are a new class of recently developed chemicals with singular characteristics that arise from their specific structure, and consequently have attracted attention from chemists. They are liquids composed not of molecules but ions, an organic cation and an organic or inorganic anion. As they are composed of charged units, they have low vapour pressures and are considered not volatile. Usually ILs are viscous liquids highly prized for their ability as solvents of not only organic but also inorganic substances. They can be miscible, immiscible or partially miscible with water, depending on the cation or the anion, and they can be tailored to have the appropriate solvent properties by introducing changes in the chemical structure of the cation or the anion $(1,2)$.

ILs have been the object of intense investigation towards uncountable chemical and biochemical applications (3). The antimicrobial activity of the first ionic liquids prepared has already been described, as well as their ecotoxicity $\left(4^{\mathrm{a}, \mathrm{b}}\right)$.

In this work, is described the synthesis of ionic liquids obtained from a 2-mercaptobenzimidazole, 2-mercaptobenzoxazole or 2-mercaptobenzothiazole anion and 1-alkyl-3-methylimidazolium or choline cation. The antimicrobial activity of these liquids against several Gram-positive and Gram-negative bacteria and a yeast was also evaluated.

\section{Materials and Methods}

\section{Materials and Equipment}

Reagents and solvents of analytical purity were provided by Sigma-Aldrich.

${ }^{1} \mathrm{H}$ NMR spectra were acquired in a Bruker Advance 400 apparatus at $400 \mathrm{MHz}$, using $\mathrm{D}_{2} \mathrm{O}, \mathrm{CDCl}_{3}$ or DM$\mathrm{SO}-\mathrm{d} 6$ as solvents. The chemical shifts are reported in parts per million (ppm, $\delta$ ), using the appropriate signal for residual solvent protons as reference. The growth of microorganisms was measured with an Absorvance Microplate Reader set to $620 \mathrm{~nm}$ (Thermo Scientific Multiskan FC).

\section{Synthesis of ionic liquids}

Synthesis of 1-alkyl-3-methylimidazolium bromide: [Cnmim $] \mathrm{Br}$

Halogenated ionic liquids, 1-ethyl-3-methylimidazolium bromide [C2mim] [Br],

\section{Introdução}

Os Líquidos Iónicos (LI) são uma nova classe de compostos químicos, recentemente desenvolvida, que possui características singulares resultantes da sua estrutura específica e que, por esse motivo, têm sido objecto de muita atenção. Estes novos materiais são líquidos constituídos não por moléculas, mas por iões: um catião orgânico e um anião que pode ser orgânico ou inorgânico. Uma vez que são constituídos por unidades carregadas, têm baixas pressões de vapor e são considerados não voláteis. Normalmente, os LI são líquidos viscosos muito apreciados pela sua capacidade como solventes, não só de substâncias inorgânicas, mas também de substâncias orgânicas. Podem ser miscíveis, imiscíveis ou parcialmente miscíveis com água, dependendo do catião e/ou anião e podem ser modificados para ter as propriedades solventes adequadas, introduzindo alterações na estrutura química do catião e/ou do anião $(1,2)$.

Os LI têm sido objeto de intensa investigação com o fim de se conhecerem as suas potencialidades em inúmeras aplicações químicas e bioquímicas (3). A atividade antimicrobiana dos primeiros líquidos iónicos já foi demonstrada, bem como a sua ecotoxicidade $\left(4^{\mathrm{a}, \mathrm{b}}\right)$. Neste trabalho, é descrita a síntese de líquidos iónicos obtidos a partir dos aniões 2-mercapto- benzimidazol, 2-mercaptobenzoxazol ou 2-mercaptobenzotiazol e dos catiões 1-alquil-3-metilimidazólio e colina. A sua atividade antimicrobiana contra diversas bactérias Gramnegativas e Gram-positivas também foi avaliada.

\section{Materiais e Métodos}

\section{Materiais e Equipamento}

Os reagentes e solventes usados, de grau analítico, foram fornecidos pela Sigma-Aldrich.

Os espectros de ${ }^{1} \mathrm{H}$ NMR foram obtidos num aparelho Brucker Avance 400, a $400 \mathrm{MHz}$, usando $\mathrm{D}_{2} \mathrm{O}, \mathrm{CDCl}_{3}$ ou DMSO-d6 como solventes. Os desvios químicos são referidos em partes por milhão (ppm, $\delta$ ), utilizando como referência o sinal apropriado do protão residual do solvente deuterado. O crescimento microbiano foi medido com um leitor de microplacas ao comprimento de onda de $620 \mathrm{~nm}$ (Termo Scientific Multiskan FC).

\section{Síntese dos Líquidos Iónicos}

Síntese do brometo de 1-alquil-3-metilimidazólio: [Cn$\underline{\operatorname{mim}] \mathrm{Br}}$

Os LI halogenados, brometo de 1-etil-3-metilimidazólio [C2mim][Br], brometo de 1-butil-3-metilimidazólio 
1-butyl-3-methylimidazolium bromide [C4mim] $[\mathrm{Br}]$ and 1-hexyl-3-methylimidazolium bromide [C6mim] $[\mathrm{Br}]$ were prepared according to the procedures described in literature $(5,6)$ with small modifications: bromoalkane (ethyl, butyl or hexyl) $(0.08 \mathrm{~mol})$ was added slowly for 1.5 hours to 1 -methylimidazole $(6 \mathrm{~g}$, $0.073 \mathrm{~mol}, 5.82 \mathrm{~mL}$ ). The mixture was stirred for 1 hour for ethyl bromide and 0.5 hour for butyl and hexyl bromide at room temperature (RT) until two phases appeared. Then the reaction mixture was heated, with constant stirring, in a water bath at $70^{\circ} \mathrm{C}$, overnight, for the ethyl derivative, and for 8 hours for the butyl and hexyl derivatives. The mixture was concentrated on a rotatory evaporator, and then washed three times with tetrahydrofuran (THF). Compounds were characterized by ${ }^{1} \mathrm{H}$ NMR and data obtained were in accordance with those reported in the literature $(5,6)$.

Synthesis of 1-alkyl-3-methylimidazolium hydroxide: [Cnmim] $] \mathrm{OH}$

Synthesis of 1-alkyl-3-methylimidazolium hydroxide was performed as previously described (6) with the following modification: the corresponding 1-alkyl-3-methylimidazolium bromide $(0.0731 \mathrm{~mol})$ was dissolved in methanol $(\mathrm{MeOH})$ and then an equimolar amount of potassium hydroxide $(\mathrm{KOH})(0.0731 \mathrm{~mol}, 4.10 \mathrm{~g})$ was added. The mixture was heated for 18 hours, in an oil bath at $60{ }^{\circ} \mathrm{C}$. The resulting potassium bromide $(\mathrm{KBr})$ was then filtered, and the $\mathrm{MeOH}$ was evaporated under vacuum. The product was used immediately in the next step without further purification.

Synthesis of 1-alkyl-3-methylimidazolium 2-mercaptobenzoimidazolide [Cnmim][TBI], 2-mercaptobenzoxazolide [Cnmim][TBO] and 2-mercaptobenzothiazolide (Cnmim][TBT] ionic liquids

ILs were prepared by neutralization of the corresponding 1-alkyl-3-methylimidazolium hydroxide. 1-ethyl3-methylimidazolium hydroxide [C2mim] $[\mathrm{OH}]$, 1-butyl-3-methylimidazolium hydroxide $[\mathrm{C} 4 \mathrm{mim}][\mathrm{OH}]$ or 1-hexyl-3-methylimidazolium hydroxide [C6mim] $[\mathrm{OH}](0.016 \mathrm{~mol})$ were dissolved in $\mathrm{MeOH}$ and an equivalent amount $(0.016 \mathrm{~mol})$ of the corresponding mercapto compound, 2-mercaptobenzimidazole (TBI), 2-mercaptobenzoxazole (TBO) or 2-mercaptobenzotiazole (TBT), was added slowly. The mixture was stirred overnight at room temperature and was then refluxed 10 hours. Evaporation of $\mathrm{MeOH}$ under vacuum afforded the corresponding IL (Table 1).

Synthesis of cholinium 2-mercaptobenzoimidazolide (ChTBI), 2-mercaptobenzoxazolide (ChTBO) and 2-mercaptobenzothiazolide (ChTBT) ionic liquids

Cholinium 2-mercaptobenzoimidazolide, 2-mercapto-
[C4mim] $[\mathrm{Br}]$ e o brometo de 1-hexil-3-metilimidazólio [C6mim][Br] foram preparados de acordo com os procedimentos descritos na literatura $(5,6)$ com pequenas modificações: adicionou-se lentamente, durante $1,5 \mathrm{~h}$, o bromoalcano (etilo, butilo ou hexilo) $(0,08 \mathrm{~mol})$ ao 1-metilimidazol (6 g, 0,073 mol, 5,82 mL). A mistura foi agitada à temperatura ambiente, durante $1 \mathrm{~h}$ na síntese do brometo de etilo e, durante $0,5 \mathrm{~h}$, nas sínteses do brometo de butilo e brometo de hexilo, até ao aparecimento de duas fases. De seguida, a mistura reacional foi aquecida, sempre sob agitação, num banho de água a $70{ }^{\circ} \mathrm{C}$, de um dia para o outro, para o derivado de etilo e, durante $8 \mathrm{~h}$, para os derivados de butilo e hexilo. A mistura foi concentrada num evaporador rotativo e, em seguida, lavada por 3 vezes com tetrahidrofurano (THF). Os compostos foram caracterizados por espectroscopia de Ressonância Magnética Nuclear de protão ( ${ }^{1} \mathrm{H}$ NMR) estando os valores obtidos de acordo com os descritos na literatura $(5,6)$.

Síntese do hidróxido de 1-alquil-3-metilimidazólio: [Cnmim] OH

A síntese do hidróxido de 1-alquil-3-metilimidazólio foi realizada de acordo com o descrito anteriormente (6), com a seguinte modificação: o brometo de 1-alquil3-metilimidazólio $(0,0731 \mathrm{~mol})$ foi dissolvido em metanol $(\mathrm{MeOH})$ e, posteriormente, foi adicionada uma quantidade equimolar de hidróxido de potássio $(\mathrm{KOH})(0,0731 \mathrm{~mol}, 4,10 \mathrm{~g})$. A mistura foi aquecida durante $18 \mathrm{~h}$ num banho de óleo a $60{ }^{\circ} \mathrm{C}$. De seguida, o brometo de potássio $(\mathrm{KBr})$ resultante foi filtrado e o $\mathrm{MeOH}$ evaporado sob vácuo. $\mathrm{O}$ produto foi utilizado imediatamente sem purificação adicional.

Síntese dos LI de 1-alquil-3-metilimidazólio e de 2-mercaptobenzoimidazolido [Cnmim][TBI], 2-mercaptobenzoxazolido [Cnmim][TBO] e 2-mercaptobenzotiazolido [Cnmim][TBT]

Os LI foram preparados por neutralização do correspondente hidróxido do 1-alquil-3-metil imidazólio. $\mathrm{O}$ hidróxido de 1-etil-3-metilimidazol [C2mim][OH], hidróxido de 1-butil-3-metilimidazol [C4mim][OH] ou hidróxido de 1-hexil-3-metilimidazol [C6mim] $[\mathrm{OH}]$ $(0,016 \mathrm{~mol})$ foram dissolvidos em $\mathrm{MeOH}$. À respetiva solução foi adicionada lentamente uma quantidade equivalente $(0,016 \mathrm{~mol})$ do composto mercapto correspondente, o 2-mercaptobenzoimidazol (TBI), o 2-mercaptobenzoxazol (TBO) ou o 2-mercaptobenzotiazol (TBT). A mistura reaccional foi agitada durante a noite à temperatura ambiente e, em seguida, foi aquecida a refluxo durante $10 \mathrm{~h}$. O líquido iónico correspondente foi obtido após evaporação do $\mathrm{MeOH}$ (Tabela 1). 
benzoxazolide and 2-mercaptobenzothiazolide compounds were prepared by neutralization of choline hydroxide by 2-mercaptobenzoimidazol (TBI), 2-mercaptobenzoxazol (TBO) and 2-mercaptobenzothiazol (TBT). 2-mercaptobenzothiazole, 2-mercaptobenzoxazole and 2-mercaptobenzimidazole were mixed with an equimolar amount of choline $(45 \% \mathrm{MeOH}$ solution) $(0.006 \mathrm{~mol})$ and stirred overnight at room temperature. The $\mathrm{MeOH}$ was subsequently evaporated and the oil residue obtained was washed with acetonitrile and dried under vacuum. IL containing 2-mercaptobenzothiazole was a dense orange oil. ILs containing 2-mercaptobenzimidazole and 2-mercaptobenzoxazole were solids with low melting points: $115^{\circ} \mathrm{C}-118{ }^{\circ} \mathrm{C}$ and $50{ }^{\circ} \mathrm{C}-70^{\circ} \mathrm{C}$, respectively (Table 1). ILs were characterized by ${ }^{1} \mathrm{H}$ NMR (Table 2 ).

\section{Antimicrobial activity}

\section{Microbial strains}

The in vitro antimicrobial study was carried out using Gram-positive bacteria (Staphylococcus aureus ATCC 25923, Enterococcus faecalis ATCC 29212, Enterococcus hirae ATCC 10541, Bacillus subtillis ATCC 6633, and Mycobacterium smegmatis ATCC 607), Gram-negative bacteria (Escherichia coli ATCC 25922, Pseudomonas aeruginosa ATCC 27853 and Klebsiella pneumoniae ATCC 9997) and a yeast (Candida albicans ATCC 10231).

\section{Well diffusion test}

The well diffusion assay was used to determine the antimicrobial activity of the compounds (7). Petri dishes containing $20 \mathrm{~mL}$ Mueller-Hinton culture medium were inoculated with $0.1 \mathrm{~mL}$ of a bacterial cell suspension matching a $0.5 \mathrm{McF}$ arland standard solution. The suspension was uniformly spread using a sterile swab over the surface of the medium. Wells of $5 \mathrm{~mm}$ in diameter were made in the agar plates with a sterile glass Pasteur pipette and $50 \mu \mathrm{L}$ of each compound $(1 \mathrm{mg} / \mathrm{mL})$, previously reconstituted by dissolving in dimethyl sulfoxide (DMSO), were added into the well. DMSO was used as a negative control, while vancomycin, norfloxacin, and amphotericin B at $1 \mathrm{mg} / \mathrm{mL}$ were used as positive controls for Gram positive and Gram-negative bacteria and the yeast, respectively. The plates were then incubated at $37{ }^{\circ} \mathrm{C}$ for 24 hours. The antimicrobial activity was assayed by measuring the diameter of the inhibition zone (in $\mathrm{mm}$ ) formed around the wells. Each assay was performed in triplicate, at minimum.

Microdilution method

The minimum inhibitory concentrations (MICs) of compounds were determined by means of the two-fold
Síntese dos líquidos iónicos de colina e de 2-mercaptobenzoimidazolido (ChTBI), 2-mercaptobenzoxazolido (ChTBO) e 2-mercaptobenzotiazolido (ChTBT)

Os compostos de colina e de 2-mercaptobenzoimidazolido, 2-mercaptobenzoxazolido e 2-mercaptobenzotiazolido foram preparados por neutralização do hidróxido de colina pelo 2-mercaptobenzoimidazol (TBI), 2-mercaptobenzoxazol (TBO) e 2-mercaptobenzotiazol (TBT). O composto mercapto correspondente foi adicionado a uma solução a $45 \%$ de colina em metanol $(0,006 \mathrm{~mol})$ e a mistura reaccional ficou sob agitação de um dia para o outro à temperatura ambiente (TA). Posteriormente, o metanol $(\mathrm{MeOH})$ foi evaporado e o resíduo oleoso foi lavado com acetonitrilo e seco sob vácuo. O líquido iónico com 2-mercaptobenzotiazol apresentou-se como um óleo denso e alaranjado. Os LI contendo 2-mercaptobenzimidazol e 2-mercaptobenzoxazole são sólidos com baixo ponto de fusão: $115^{\circ} \mathrm{C}-$ $118{ }^{\circ} \mathrm{C}$ e $50{ }^{\circ} \mathrm{C}-70{ }^{\circ} \mathrm{C}$, respetivamente (Tabela 1). Os LI foram caracterizados por ${ }^{1} \mathrm{H}$ RMN (Tabela 2).

\section{Actividade antimicrobiana}

\section{Estirpes microbianas}

A avaliação da actividade antimicrobiana foi realizada in vitro, usando bactérias Gram-positivas (Staphylococcus aureus ATCC 25923, Enterococcus faecalis ATCC 29212, Enterococcus hirae ATCC 10541, Bacillus subtilis ATCC 6633, e Mycobacterium smegmatis ATCC 607), bactérias Gram-negativas (Escherichia coli ATCC 25922, Pseudomonas aeruginosa, ATCC 27853), Klebsiella pneumoniae ATCC 9997) e uma levedura (Candida albicans ATCC 10231).

\section{Teste de difusão em poço}

O ensaio de difusão foi utilizado para determinar a actividade antimicrobiana dos compostos (7). As placas de Petri, contendo $20 \mathrm{~mL}$ de meio de cultura MuellerHinton, foram inoculados com $0,1 \mathrm{~mL}$ de uma suspensão bacteriana correspondendo a uma solução padrão de 0,5 McFarland. A suspensão foi uniformemente espalhada utilizando uma zaragatoa estéril sobre a superfície do meio. Poços de $5 \mathrm{~mm}$ de diâmetro foram feitos em placas de agar com uma pipeta de Pasteur de vidro estéril e $50 \mu \mathrm{L}$ de cada composto $(1 \mathrm{mg} / \mathrm{mL})$, previamente reconstituídos por dissolução em dimetilsulfóxido (DMSO), foram adicionados a cada poço. O solvente DMSO foi usado como controlo negativo, enquanto a vancomicina, norfloxacina e anfotericina $B$, a $1 \mathrm{mg} /$ $\mathrm{mL}$, foram utilizados como controlos positivos para as bactérias Gram-positivas, Gram-negativas e a levedura, respectivamente. As placas foram então incubadas a 37 ${ }^{\circ} \mathrm{C}$ durante 24 horas. A actividade antimicrobiana foi determinada medindo o diâmetro da zona de inibição (em $\mathrm{mm}$ ) formada à volta dos poços. Cada ensaio foi efectuado pelo menos em triplicado.

Método de microdiluição 
serial broth microdilution assay (7). The compounds, dissolved in DMSO, were diluted with Mueller-Hinton broth medium to concentrations ranging from $500 \mu \mathrm{g} /$ $\mathrm{mL}$ to $0.488 \mu \mathrm{g} / \mathrm{mL}$. The antimicrobial activity of the solvent was also evaluated. Vancomycin, rifampicin and amphotericin B were used as controls. The MIC values were taken as the lowest concentration of the compound, in $\mu \mathrm{g} / \mathrm{mL}$, that inhibited the growth of the microorganisms following 24 hours of incubation at 37 ${ }^{\circ} \mathrm{C}$. Assays were carried out in triplicate for each tested microorganism.

\section{Results and Discussion}

Eleven ionic liquids were prepared (Figure 1) with a good purity level. Some of these compounds, those obtained from 2-mercaptobenzimidazole (TBI), 2-mercaptobenzoxazole (TBO) and 2-mercaptobenzothiazole (TBT), are here described for the first time.

All ionic liquids were synthesized in quantitative yield, with the exception of the 1-alkyl-3-methylimidazolium bromides, which were obtained with a lower yield (62 $\%-67 \%$ ). Color and physical state at room temperature are described in Table 1.
As concentrações mínimas inibitórias (CMI) dos compostos foram determinadas por um ensaio de microdiluição de 1:2 em série (7). Os compostos, dissolvidos em DMSO, foram diluídos em concentrações de $500 \mu \mathrm{g} / \mathrm{mL}$ - 0,488 $\mu \mathrm{g} / \mathrm{mL}$, com meio de cultura Mueller-Hinton. A atividade antimicrobiana do solvente foi avaliada e os fármacos vancomicina, rifampicina e anfotericina B utilizados como controlo. Os valores das CMI foram determinados como a concentração mais baixa do composto que inibiu o crescimento dos microrganismos, após $24 \mathrm{~h}$ de incubação a $37^{\circ} \mathrm{C}$ e são apresentados em $\mu \mathrm{g} / \mathrm{mL}$. Os ensaios foram realizados em triplicado para cada microrganismo testado.

\section{Resultados e Discussão}

No decorrer deste estudo, foram obtidos onze líquidos iónicos (Figura 1), com bom grau de pureza. Alguns destes compostos, os obtidos a partir de 2-mercaptobenzoimidazol (TBI), 2-mercaptobenzoxazol (TBO) e 2-mercaptobenzotiazol (TBT) são aqui descritos pela primeira vez.

Todos os líquidos iónicos foram sintetizados com um rendimento quantitativo, à exceção dos brometos de 1-alquil3-metilimidazólio que foram obtidos com um rendimento mais baixo, entre $62 \%$ - $67 \%$. O estado físico à temperatura ambiente dos LI sintetizados, bem como a sua cor, são descritos na Tabela 1.
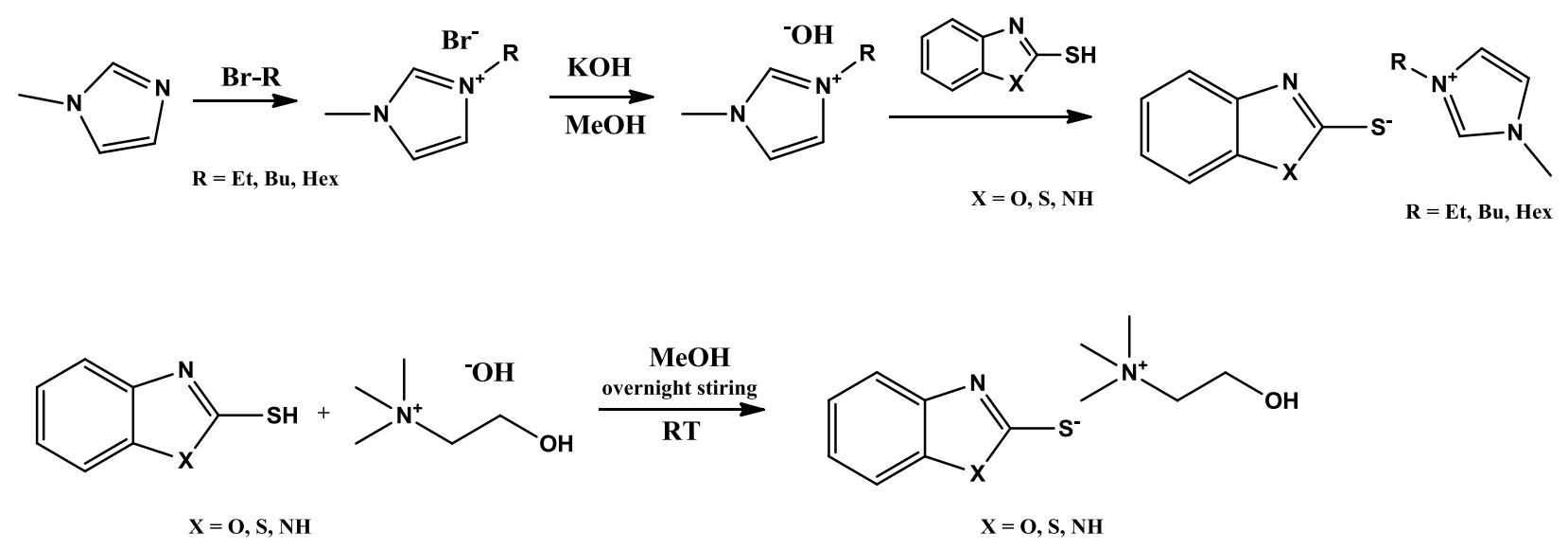

Figure 1/ Figura 1: Schematic synthesis of the new ionic liquids

Esquema de síntese dos novos líquidos iónicos

Characterization of the new compounds was performed by ${ }^{1} \mathrm{H}$ NMR and spectral data are presented in Table 2. The antimicrobial activity of the prepared ionic liquids was investigated by the well diffusion assay. Compounds were tested against five Gram-positive bacteria (Staphylococcus aureus, Enterococcus faecalis, Enterococcus hirae, Bacillus subtillis and Mycobacterium smegmatis), three Gram-negative bacteria (Escherichia
A caracterização dos novos compostos foi realizada por ${ }^{1} \mathrm{H}$ RMN e os dados espectrais são apresentados na Tabela 2.

A atividade antimicrobiana dos líquidos iónicos sintetizados foi investigada através do ensaio de difusão em poço. Este teste foi utilizado como um rastreio para determinar a capacidade destes compostos para inibir 
Table 1/ Tabela 1. Physical state and color of the synthesized new ionic liquids

Estado físico e cor dos novos líquidos iónicos sintetizados

\begin{tabular}{lcc}
\hline $\begin{array}{l}\text { Ionic Liquid/ } \\
\text { Líquido Iónico }\end{array}$ & $\begin{array}{c}\text { Physical state at RT } \\
\text { Estado Físico à TA }\end{array}$ & Color/ Cor \\
\hline$[\mathrm{C} 2 \mathrm{mim}][\mathrm{Br}]$ & Solid/Sólido & White/Branco \\
{$[\mathrm{C} 4 \mathrm{mim}][\mathrm{Br}]$} & Solid/Sólido & Light yellow/Amarelo claro \\
{$[\mathrm{C} 6 \mathrm{mim}][\mathrm{Br}]$} & Liquid/Líquido & Light yellow/Amarelo claro \\
{$[\mathrm{C} 2 \mathrm{mim}][\mathrm{TBI}]$} & Solid/Sólido & White/Branco \\
{$[\mathrm{C} 4 \mathrm{mim}][\mathrm{TBO}]$} & Liquid/Líquido & Light Brown/Castanho claro \\
{$[\mathrm{C} 4 \mathrm{mim}][\mathrm{TBT}]$} & Solid/Sólido & Yellow/Amarelo \\
{$[\mathrm{C} 6 \mathrm{mim}][\mathrm{TBO}]$} & Liquid/Líquido & Brown/Castanho \\
{$[\mathrm{C} 6 \mathrm{mim}][\mathrm{TBT}]$} & Liquid/Líquido & Brown/Castanho \\
{$[\mathrm{Ch}][\mathrm{TBI}]$} & Solid/Sólido & Beige/Bege \\
{$[\mathrm{Ch}][\mathrm{TBO}]$} & Solid/Sólido & Light Brown/Castanho claro \\
{$[\mathrm{Ch}][\mathrm{TBT}]$} & Liquid/Líquido & Light Orange/Laranja claro \\
\hline
\end{tabular}

coli, Pseudomonas aeruginosa and Klebsiella pneumoniae), and a yeast (Candida albicans).

Compounds [C4mim][TBO], [C6mim][TBT] and $[\mathrm{C} 2 \mathrm{mim}][\mathrm{TBI}]$ showed inhibition zones against Gram-positive B. subtillis ( $8 \mathrm{~mm}$ of inhibition zone for compound [C6mim][TBT] and $P$. aeruginosa (9 $\mathrm{mm}$ inhibition zone for both compounds [C4mim] $[\mathrm{TBO}]$ and $[\mathrm{C} 2 \mathrm{mim}][\mathrm{TBI}]$. The remaining imidazolium compounds as well as the choline based ionic liquids showed no inhibition zones, as did the DMSO solvent, against all the bacteria and the yeast tested (Table 3 ). The described positive results for compounds [C4mim] [TBO], [C6mim][TBT] and [C2mim][TBI] were further investigated by the microdilution method. Compounds [C6mim $][\mathrm{TBT}]$ and $[\mathrm{C} 2 \mathrm{mim}][\mathrm{TBI}]$ showed MIC values of $62.5 \mu \mathrm{g} / \mathrm{mL}$ against the Gram-positive $B$. subtillis and Gram-negative P.aeruginosa bacteria, thus revealing modest antibacterial activity. Antimicrobial activity of these compounds is of the same magnitude as the activities of other ILs (8).

Choline based ionic liquids, [Ch][TBO], [Ch][TBT] and $[\mathrm{Ch}][\mathrm{TBI}]$ as well as the imidazolium based ionic liquids, $[\mathrm{C} 4 \mathrm{mim}][\mathrm{Br}],[\mathrm{C} 4 \mathrm{mim}][\mathrm{TBT}]$ and $[\mathrm{C} 6 \mathrm{mim}]$ [TBO] did not exhibit any antimicrobial activity.

In literature $(1,4,9)$, it is indicated that three substructures of an ionic liquid have importance in the evaluation of their toxicity against several organisms, including bacteria, algae, plants and invertebrates: a) a o crescimento de bactérias, quer Gram-positivas, quer Gram-negativas e também de uma levedura.

Os compostos foram testados contra cinco bactérias Grampositivas (Staphylococcus aureus, Enterococcus faecalis, Enterococcus hirae, Bacillus subtilis e Mycobacterium smegmatis), três bactérias Gram-negativas (Escherichia coli, Pseudomonas aeruginosa e Klebsiella pneumoniae), e uma levedura (Candida albicans). Os compostos [C4mim][TBO], [C6mim][TBT] e [C2mim][TBI] revelaram zonas de inibição contra a bactéria Gram-positiva B. subtillis $(8 \mathrm{~mm}$ de zona de inibição para o composto [C6mim][TBT]) e P. aeruginosa $(9 \mathrm{~mm}$ de zona de inibição para ambos os compostos [C4mim][TBO] e [C2mim][TBI]). Os restantes compostos de imidazólio, bem como os compostos de colina não indicaram zonas de inibição (tal como o solvente DMSO) contra todas as bactérias Gram-positivas, Gram-negativas e a levedura testadas (Tabela 3). Os resultados positivos descritos anteriormente para os compostos [C4mim][TBO], [C6mim] [TBT] e [C2mim][TBI] foram posteriormente estudados pelo método de microdiluição. Os compostos [C6mim][TBT] e [C2mim][TBI] revelaram valores de CMI de $62,5 \mu \mathrm{g} / \mathrm{mL}$ contra as bactérias Gram-positiva B. subtillis e Gram-negativa P.aeruginosa, indicando assim uma atividade antibacteriana moderada. A actividade antimicrobiana destes compostos é da mesma ordem de grandeza das atividades de outros LI descritos na literatura (8).

Os líquidos iónicos contendo o catião colina [Ch][TBO], [Ch] [TBT] e [Ch] [TBI] não apresentaram qualquer atividade antimicrobiana. Da mesma forma, os líquidos iónicos contendo o catião alquil-metilimidazólio, [C4mim] Br, [C4mim][TBT] 
Table 2/ Tabela 2 - 1H NMR data of the synthesized ionic liquids

Dados de 1H RMN dos líquidos iónicos sintetizados



* multiplicity: s, singlet; d, duplet; dd, doble duplet; t, triplet; q, quartet; qt, quintet; st, sextet; m, multiplet. Deuterated solvent:, ${ }^{\mathrm{a}} \mathrm{CDCl}{ }_{3}$, ${ }^{\mathrm{b}} \mathrm{DMSO}-\mathrm{d} 6,{ }^{\mathrm{c}} \mathrm{D}_{2} \mathrm{O} .{ }^{1}$ and ${ }^{2}$ can be inter-changeable

* multiplicidade: s, singuleto; d, dubleto; dd, dubleto duplo; t, tripleto; q, quarteto; qt, quinteto; st sexteto; m, multipleto. Solvente deuterado: ${ }^{\mathrm{a}} \mathrm{CDCl}{ }_{3},{ }^{\mathrm{b}} \mathrm{DMSO}-\mathrm{d} 6,{ }^{\mathrm{c}} \mathrm{D}_{2} \mathrm{O}$. ${ }^{1} \mathrm{e}^{2}{ }^{2}$ podem ser interalteradas

positive portion designated as head-group, b) the substituents present in that head-group, and c) the anion. Table 3 shows that a small inhibition in the growth of the Gram-positive bacteria was obtained with [C6mim] [TBT], [C4mim][TBO] and [C2mim][TBI], and these were also slightly active against $P$. aeruginosa. It is interesting to note that regarding the bacteria tested, changing the head group from an alkyl imidazolium to choline removed the antibacterial effect, which could indicate that the head group was the responsible for this activity. However, the lack of activity of [C4mim] $\mathrm{Br}$ and $[\mathrm{C} 4 \mathrm{mim}][\mathrm{TBT}]$ does not support this hypothesis. It is also stated $(9,10)$ that the length of the alkyl chain attached to the head group influences the toxicity of the ionic liquid - the longer the chain, the more active the ionic liquid. It was thus expected that [C6mim] [TBO] would be more active than the corresponding IL, [C4mim][TBO], which contains a smaller alkyl chain linked to the same head group, but this was not observed. In a previous work (6), it was also found that toxicity against bacteria does not follow the same rules previously found in organisms with a higher degree of organization. Different susceptibility of Gram-positive and Gram-negative bacteria to the same IL is likely due to the different compositions of the cell walls. e [C6mim][TBO], também não foram ativos contra os microorganismos testados.

Na literatura $(1,4,9)$, é referido que três sub-estruturas de um líquido iónico têm importância para a avaliação da sua toxicidade contra organismos diversos como bactérias, algas, plantas e invertebrados: a) porção positiva, ou catião, designada como grupo-cabeça, b) os substituintes presentes no grupo-cabeça e c) 0 anião.

A Tabela 3 mostra que uma pequena inibição do crescimento das bactéria Gram-positiva B. subtilis, foi obtida com [C6mim][TBT]. Os compostos [C4mim][TBO] e [C2mim] $[\mathrm{TBI}]$ também foram ligeiramente ativos contra $P$. aeruginosa. É interessante observar que em relação às bactérias testadas, a mudança do grupo positivo de um alquil-metilimidazólio para a colina, retira o efeito antibacteriano, o que pode indicar ser este o responsável por essa atividade. No entanto, a falta de atividade de [C4mim]Br e [C4mim][TBT] não apoia esta hipótese. Também é afirmado $(9,10)$ que o comprimento da cadeia alquílica pertencente ao catião influencia a toxicidade do líquido iónico, isto é, quanto maior for a cadeia, mais ativo é o LI. Assim, esperava-se que [C6mim][TBO] fosse mais ativo do que o LI correspondente, [C4mim][TBO], que contém uma menor cadeia alquílica, mas isso não se verificou. Num trabalho anterior (6), também se demostrou que a toxicidade contra bactérias não segue as mesmas regras já encontradas em organismos com maior grau de organização. A diferente suscetibilidade de bactérias Gram-positivas e Gramnegativas para o mesmo LI é, provavelmente, devida a diferenças na composição da parede celular. 
Table 3/ Tabela 3: Antibacterial activity of ILs

Atividade antibacteriana dos LI

\begin{tabular}{|c|c|c|c|c|}
\hline \multirow{2}{*}{$\begin{array}{l}\text { Compound } \\
\text { Composto }\end{array}$} & \multicolumn{2}{|c|}{$\begin{array}{l}\text { Inhibition zone/Zona de inibição(mm) } \\
\qquad(C=1 \mathrm{mg} / \mathrm{mL})\end{array}$} & \multicolumn{2}{|c|}{$\begin{array}{c}M I C / C M I \\
(\mu \mathrm{g} / \mathrm{mL})\end{array}$} \\
\hline & B. subtilis & P. aeruginosa & B. subtilis & P. aeruginosa \\
\hline$[\mathrm{C} 4 \mathrm{mim}][\mathrm{TBO}]$ & $-*$ & 9 & $\mathrm{nt}$ & $>125$ \\
\hline$[\mathrm{C} 6 \mathrm{mim}][\mathrm{TBT}]$ & 8 & $-*$ & 62,5 & nt \\
\hline$[\mathrm{C} 2 \mathrm{mim}][\mathrm{TBI}]$ & $-*$ & 9 & $\mathrm{nt}$ & 62,5 \\
\hline TBT & 9 & 8 & 250 & 62,5 \\
\hline TBI & $-*$ & 8 & nt & 62,5 \\
\hline TBO & $-*$ & 7 & $\mathrm{nt}$ & 62,5 \\
\hline Positive control** & 27 & 35 & $<7,82$ & $<7,82$ \\
\hline DMSO & $-*$ & $-*$ & 125 & 125 \\
\hline
\end{tabular}

* no inhibition; **Gram + bacteria: Vancomycin, Gram -: Norfloxacyn; nt, not tested

* sem inibição; **Bactérias Gram + : Vancomicina, Gram -: Norfloxacina; nt, não testado

\section{Conclusion}

In conclusion, a new approach for the synthesis of benzoazole ionic liquids has been developed.

The resulting compounds have been tested for antibacterial and antifungal activities. The present series of compounds exhibited moderate antimicrobial activity against Gram-positive and Gram-negative bacteria, which opens the avenue to use these compounds as chemotherapeutic agents. Further studies on the synthesis of a library of ionic liquids and evaluation of their antimicrobial activity must be performed, as the antimicrobial activity of this huge class of chemicals is difficult to predict and is an enormous challenge to chemists and microbiologists.

\section{Acknowledgments}

The authors are thankful to Professor Aida Duarte (Faculdade de Farmácia da Universidade de Lisboa, Lisboa, Portugal) for providing the microorganisms tested. The funding from FCT (Fundação para a Ciência e a Tecnologia, Portugal) is also gratefully acknowledged (Project PEst-OE/QUI/UI0612/2013).

\section{Conflict of Interest}

The authors declare that there is no financial or personal relationship that can be understood as representing a potential conflict of interest.

\section{Conclusão}

Como conclusão, pode afirmar-se que foi desenvolvida uma nova abordagem para a síntese de líquidos iónicos baseados nos heterocíclos do tipo benzoazol, os quais podiam conter o átomo de azoto, oxigénio ou enxofre. Os compostos resultantes foram testados quanto à atividade anti-bacteriana e anti-fúngica. A presente série de compostos exibiu alguma atividade antimicrobiana contra bactérias Gram-positivas e Gram-negativas, o que abre o caminho para a utilização destes compostos como agentes terapêuticos. A síntese/elaboração de uma biblioteca de líquidos iónicos e avaliação da sua atividade antimicrobiana é uma área de pesquisa científica que deve ser continuada uma vez que, para estes compostos, esta atividade é difícil de prever, mostrando-se um enorme desafio para os químicos e microbiologistas

\section{Agradecimentos}

Os autores agradecem à Professora Aida Duarte (Faculdade de Farmácia da Universidade de Lisboa, Lisboa, Portugal) por ceder os microrganismos utilizados neste trabalho.

Este trabalho foi financiado pela Fundação para a Ciência e a Tecnologia (Portugal) através do Projecto PEstOE/QUI/UI0612/2013.

\section{Conflito de interesses}

Os autores declaram que não há nenhuma relação financeira ou pessoal, que possa ser entendida como representando um potencial conflito de interesses. 


\section{References / Referências}

[1] Brennecke JFD, Rogers RD, Seddon KR. (Eds.). Ionic liquids IV: not just solvents anymore, ACS Symposium Series, American Chemical Society, Washington; 2007.

[2] Kokorin A (ed.). Ionic liquids: Applications and perspectives. InTech 2011, 674p. (Accessed April 2013 at http://www.intechopen. $\mathrm{com} /$ books/ionic-liquids-applications-andperspectives).

[3] Jain N, Kumar A, Chauhan S, Chauan SMS. Chemical and biochemical transformations in ionic liquids. Tetrahedron 2005; 6: 1015 1060.

[4] a Pham TPT, Cho CW, Yun YS. Environmental fate and toxicity of ionic liquids: a review. Water Research 2010; 44: 352-372.; b Garcia MT, Ribosa I, Perez L, Manresa A, Comelles F. Aggregation Behavior and Antimicrobial Activity of Ester-Functionalized Imidazolium- and Pyridinium-Based Ionic Liquids in Aqueous Solution. Langmuir 2013; 29: 2536-2545.
[5] Owens GS, Abu-Omar MM. Comparative kinetic investigations in ionic liquids using the MTO/peroxide system. Journal of Molecular Catalyst A: Chemical 2002; 187: 215-225.

[6] Gouveia W, Jorge TF, Martins S, Meireles M, Carolino M, Cruz C, Almeida TS, Araújo MEM. Toxicity of ionic liquids prepared from biomaterials. Chemosphere 2014; 104:51-56

[7] CLSI- Clinical and Laboratory Standards Institute, 2011. Performance standards for antimicrobial susceptibility testing: Twenty First International Supplement M100-S21. Clinical and Laboratory Standards Institute, Wayne, PA.

[8] Khungar B, Rao M S, Pericherla K, Nehra P, Jain N, Panwar J, Kumar A. Synthesis, characterization and microbiocidal studies of novel ionic liquid tagged Schiff bases. Comptes Rendues Chimie 2012; 15: 669674.
[9] Romero A, Santos A, Tojo J, Rodríguez A. Toxicity and biodegradability of imidazolium ionic liquids. Journal of Hazardous Materials $2008 ; 151: 268-273$.

[10] Cho C-W, Pham TPT, JeonY-C, Vijayaraghavan K, ChoeW-S,Yun Y-S. Toxicity of imidazolium salt with anion bromide to a phytoplankton Selenastrum capricornutum: Effect of alkyl-chain length. Chemosphere 2007; 6: 1003-1007. 